\title{
MACROFAUNA DO SOLO INFLUENCIADA PELO USO DE FERTILIZANTES QUÍMICOS E DEJETOS DE SUÍNOS NO OESTE DO ESTADO DE SANTA CATARINA ${ }^{(1)}$
}

\author{
Maurício Vicente Alves ${ }^{(2)}$, Júlio Cesar Pires Santos ${ }^{(3)}$, Deisi Tatiani \\ de Gois ${ }^{(4)}$, Janaina Veronezi Alberton ${ }^{(5)} \&$ Dilmar Baretta ${ }^{(6)}$
}

\begin{abstract}
RESUMO
Este estudo objetivou avaliar o efeito da adição de doses crescentes de dejetos de suínos, adubação organomineral e adubação mineral sobre a abundância e diversidade da macrofauna edáfica. O estudo foi realizado no município de Campos Novos, SC, em um Latossolo Vermelho distroférrico, cultivado anualmente com milho (Zea mays) e aveia (Avena sativa), em sucessão e semeadura direta. Os seis tratamentos utilizados foram: testemunha (TT); adubação mineral (AM); adubação organomineral (AOM); adubação orgânica na doses de $3 \mathrm{t} \mathrm{ha}^{-1}$ (AO3); $6 \mathrm{t} \mathrm{ha}^{-1}$ (AO6) e $12 \mathrm{t} \mathrm{ha}^{-1}$ (AO12) de dejeto de suínos em base seca. O fertilizante mineral foi aplicado em semeadura na linha do plantio e a adubação orgânica, após semeadura do milho. A macrofauna edáfica foi avaliada utilizando-se um amostrador cilíndrico com $17 \mathrm{~cm}$ de diâmetro, coletando-se amostras de solo na profundidade de 0-10 cm. As amostragens foram realizadas em três épocas: a primeira em 5/5/2005 (um mês após a colheita do milho), a segunda em 9/09/2005 (sob a cultura de aveia), e a terceira em 23/1/2006 (sob a cultura do milho). A diversidade de organismos foi avaliada por meio dos índices de Shannon, Simpson e Pielou, riqueza de grupos, abundância e análise de agrupamento. A freqüência relativa das ordens foi afetada pela adição do dejeto suíno, sendo os grupos mais freqüentes Hymenoptera e Oligochaeta, variando conforme o tratamento e a época de amostragem. A maior
\end{abstract}

(1) Parte da Tese de Mestrado do primeiro autor. Recebido para publicação em fevereiro de 2007 e aprovado em novembro de 2007.

(2) Engenheiro-Agrônomo, Mestrando em Ciência do Solo, Departamento de Solo do Centro de Ciências Agroveterinárias, Universidade do Estado de Santa Catarina - CAV/UDESC. Av. Luiz de Camões 2090, CEP 88520-000 Lages (SC). E-mail: mauriciovicente@gmail.com

(3) Professor do Departamento de Solo do Centro de Ciências Agroveterinárias, CAV/UDESC. E-mail: a2jcps@cav.udesc.br

(4) Acadêmica do Curso de Engenharia Florestal, Departamento de Solo do Centro de Ciências Agroveterinárias, CAV/UDESC. Bolsista do PROBIC/UDESC. E-mail: deisitg@hotmail.com

(5) Acadêmica do Curso de Agronomia Departamento de Solo do Centro de Ciências Agroveterinárias, CAV/UDESC. E-mail: jvalberton@yahoo.com.br

(6) Engenheiro-Agrônomo, Doutorando do PPG em Solos e Nutrição de Plantas, Escola Superior de Agricultura "Luiz de Queiroz", Universidade de São Paulo - ESALQ/USP. Av. Pádua Dias 11, Caixa Postal 9, CEP 13418-900 Piracicaba (SP). E-mail: baretta@esalq.usp.br 
diversidade da macrofauna foi encontrada no tratamento AOM, demonstrando que a macrofauna edáfica foi influenciada pela adição consorciada das diferentes formas de adubação.

Termos de indexação: esterco de suíno, fauna edáfica, adubação mineral.

\title{
SUMMARY: SOIL MACROFAUNA AS INFLUENCED BY CHEMICAL FERTILIZERS AND SWINE MANURE USE IN WESTERN SANTA CATARINA STATE, BRAZIL
}

\begin{abstract}
The present study aimed to evaluate the effect of addition of increasing rates of swine manure, mineral fertilizers, and the combination of both on the abundance and diversity of soil macrofauna. The study was carried out in Campos Novos county, in the western Santa Catarina state, on an Oxisol cultivated with corn (Zea mays) and oat (Avena sativa) in succession and no tillage system. Six treatments were used: a control, with no fertilization (TT); mineral fertilization (AM); swine manure + mineral fertilizer (AOM); and swine

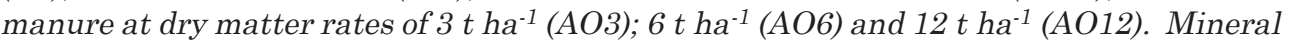
fertilizer was applied in-furrow, at sowing, and swine manure just after corn sowing. The soil macrofauna was evaluated with a cylinder of $17 \mathrm{~cm}$ diameter. Three samplings in the top layer $0-10 \mathrm{~cm}$ were carried out: the first in 5/5/2005 (one month after corn harvest), the second in 9/09/2005 (under oat) and the third in 23/1/2006 (under corn). The diversity of organism groups was evaluated by the Shannon, Simpson and Pielou indexes, group richness, abundance and cluster analysis. The relative frequency of the orders was affected by swine manure addition. Hymenoptera and Oligochaeta were the most frequent groups and they varied according to the treatment and sampling time. The greatest diversity in macrofauna was found under the AOM treatment, demonstrating that the soil macrofauna was affected by fertilization.
\end{abstract}

Index terms: edaphic fauna, mineral fertilizers, swine manure.

\section{INTRODUÇÃO}

A criação de suínos vem crescendo a cada ano, nos mercados nacional e internacional, com uma exportação de carne de 625.000 toneladas. Essa é uma atividade econômica importante, principalmente na região oeste do Estado de Santa Catarina (SC), onde se concentra o maior número de suinocultores, com rebanho de 4.465 .716 cabeças o que corresponde a $79,61 \%$ do rebanho estadual e aproximadamente $15 \%$ da produção nacional (IBGE, 2006).

A grande produção de dejetos nos confinamentos deixa ao produtor como principal alternativa o descarte desses no solo como fertilizante orgânico. Alguns estabelecimentos que possuem produção de suínos em grande escala não têm como parte de suas atividades a produção de grãos; assim, não possuem áreas adequadas para aplicação do dejeto na forma de adubação orgânica (Miner, 1999). Na região oeste de Santa Catarina, isso se agrava mais, pois há alta concentração de animais e poucas áreas agricultáveis para receber o dejeto em grande quantidade (Seiffert \& Perdomo, 1998).

Devido à alta concentração de animais e poucas áreas adequadas para aplicação dos dejetos, freqüentemente ocorre uma dosagem excessiva no solo, o que pode ocasionar acúmulo de nutrientes como $\mathrm{NO}_{3}{ }^{-}$, $\mathrm{Cu}, \mathrm{Zn}$ e de patógenos, e estes podem ser carregados por meio da enxurrada e, ou, lixiviados, podendo contaminar a água subterrânea e os mananciais (Baretta et al., 2003). Dessa forma, o dejeto suíno tem recebido considerável atenção da pesquisa agrícola, em virtude do alto risco de contaminação dos rios e do lençol freático (Scherer \& Castilhos, 1994; Baretta et al., 2003).

A adição de resíduos orgânicos em sistemas de cultivo é um fator que pode influenciar a biota do solo, principalmente pelo fornecimento de alimento para os organismos e modificações na temperatura e cobertura do solo (Correia \& Pinheiro, 1999; Baretta et al., 2003). A macrofauna do solo tem importante papel nos processos do ecossistema no que concerne à ciclagem de nutrientes e estrutura do solo, pois é responsável pela fragmentação dos resíduos orgânicos, mistura das partículas minerais e orgânicas, redistribuição da matéria orgânica, além de produzir "pellets fecais" (Hendrix et al., 1990; Baretta et al., 2007). Alterações na macrofauna edáfica podem ocorrer em função do uso da terra, de modificações no ambiente, do preparo e cultivo do solo e da adição de matéria orgânica (Baretta et al., 2003, 2006, 2007), 
ocasionando efeitos benéficos ou prejudiciais para a fauna epigéica (Alves et al., 2006).

O efeito do uso de diferentes formas de dejetos pode levar a resultados benéficos ou maléficos à macrofauna edáfica, variando conforme a composição do dejeto. Estudos realizados no oeste semi-árido da África, envolvendo esterco de gado e ovelha, palha de milho e Andropogon e composto orgânico, demonstraram que o esterco de ovelha e a palha de Andropogon foram os que mais favoreceram a macrofauna, quando comparados aos demais tratamentos (Ouédraogo et al., 2006). Alguns impactos negativos estão relacionados com a qualidade e a quantidade da matéria orgânica, a relação $\mathrm{C} / \mathrm{N}$, o aumento da compactação, podendo causar decréscimo da biomassa fresca de indivíduos e desaparecimento de espécies naquele habitat, além de alterações no funcionamento do ecossistema (Decaëns et al., 1999; Baretta et al., 2007). Portanto, o conhecimento dos grupos funcionais da macrofauna do solo de áreas utilizadas pelo homem pode fornecer informações sobre o impacto gerado no solo, a partir da exclusão de um ou mais organismos edáficos (Andersen, 1999; Baretta et al., 2007).

Este estudo objetivou avaliar o efeito da adição de doses crescentes de dejetos de suínos, adubação organomineral e adubação mineral sobre a abundância e diversidade da macrofauna edáfica de um Latossolo Vermelho distroférrico sob condições subtropicais.

\section{MATERIAL E MÉTODOS}

As amostras de solo para avaliação da macrofauna edáfica foram coletadas em uma área experimental que avalia a fertilização do solo com dejetos de suínos desde novembro de 2001, no município de Campos Novos, SC (51 ${ }^{\circ} 21$ ' 48 "W e $27^{\circ} 23$ ' 33 " S), com altitude média de $908 \mathrm{~m}$. O solo utilizado no estudo é um Latossolo Vermelho distroférrico, textura argilosa (Quadro 1), onde anualmente é cultivado em sucessão milho (Zea mays), no período estival, e aveia (Avena sativa), no período hibernal, em sistema plantio direto há seis anos.

O delineamento experimental foi constituído de blocos ao acaso, com fatorial de $6 \times 6$. As parcelas apresentam dimensões de $12 \times 6,3 \mathrm{~m}$. Os seis tratamentos testados foram: (1) testemunha (TT), não recebeu nenhum tipo de adubação; (2) adubação mineral (AM), conforme a recomendação da Comissão de Química e Fertilidade do Solo do Núcleo Regional Sul da Sociedade Brasileira de Ciência do Solo CQFSRS/SC (2004) para o local e cultivos utilizados. $\mathrm{O}$ fertilizante mineral foi aplicado junto na semeadura ao lado da semente, nas doses de $140 \mathrm{~kg} \mathrm{ha}^{-1}$ de nitrogênio (N) (40 kg ha-1 aplicados na base e $100 \mathrm{~kg} \mathrm{ha}^{-1}$ em cobertura, na forma de uréia), $70 \mathrm{~kg} \mathrm{ha}{ }^{-1}$ de fósforo (P) e $100 \mathrm{~kg} \mathrm{ha}^{-1}$ de potássio (K) por parcela; (3) adubação organomineral (AOM), com aplicação de 1,5 t ha-1 de dejetos de suínos (base seca), correspondendo a $25 \mathrm{~m}^{3} \mathrm{ha}^{-1}$ (estimada para suprir toda a recomendação de fósforo), completando-se a dose de $\mathrm{N}$ e K contida no dejeto com adubação mineral até o valor da recomendação da CQFSRS/SC (2004). O adubo mineral utilizado foi cloreto de potássio na dose de $70 \mathrm{~kg} \mathrm{ha}^{-1}$ de $\mathrm{K}$ e o $\mathrm{N}$ foi complementado em cobertura na dose de $45 \mathrm{~kg} \mathrm{ha}^{-1}$ de $\mathrm{N}$, na forma de uréia; (4) adubação orgânica na dose de $3 \mathrm{t} \mathrm{ha}^{-1}$ de dejeto de suínos (base seca), correspondendo a $50 \mathrm{~m}^{3} \mathrm{ha}^{-1}$ de dejeto (AO3); (5) adubação orgânica na dose de $6 \mathrm{t} \mathrm{ha}^{-1}$ de dejeto de suínos (base seca), correspondendo a $100 \mathrm{~m}^{3} \mathrm{ha}^{-1}$ de dejeto (AO6); (6) adubação orgânica na dose de $12 \mathrm{t} \mathrm{ha}^{-1}$ de dejeto de suínos (base seca), correspondendo a $200 \mathrm{~m}^{3} \mathrm{ha}^{-1} \mathrm{de}$ dejeto (AO12). O dejeto de suínos foi proveniente de pocilga de uma granja com animais em fase de terminação, no sistema de confinamento, com limpeza intermitente das instalações. O dejeto permaneceu armazenado em estrumeira a céu aberto por aproximadamente 40 dias antes da aplicação e foi aplicado a lanço logo após a semeadura do milho, medindo-se a dose do dejeto por meio da vazão do distribuidor em determinado período. O dejeto foi caracterizado quimicamente de acordo com o quadro 1 .

As amostras de solo para avaliação da macrofauna foram coletadas em três épocas diferentes. A primeira coleta (5/5/2005) foi realizada um mês após a colheita do milho (Zea mays), sob os restos culturais da cultura antecedente; a segunda (9/9/2005), sob a cobertura de aveia (Avena sativa) no estádio de emborrachamento; e a terceira (23/1/2006), sob a cultura do milho no estádio de enchimento de grãos. Para avaliação da macrofauna edáfica, coletou-se aleatoriamente um ponto de amostragem em cada parcela. O método utilizado foi a catação manual, descrito por Baretta (2003). Para isso, foi utilizado um tubo de aço inoxidável de $17 \mathrm{~cm}$ de diâmetro, amostrando o solo na profundidade de 0-0,10 m (Baretta, 2003). Nos mesmos pontos de amostragem da macrofauna do solo, também foram retiradas amostras para análise dos seus atributos químicos (Quadro 1), sendo analisadas conforme método descrito em Tedesco et al. (1995).

A abundância da fauna do solo nas diferentes áreas foi avaliada pelos índices de diversidade de Shannon (H), dominância de Simpson (Is) e uniformidade de Pielou (e) (Odum, 1983). A abundância da fauna do solo (organismos por $\mathrm{m}^{2}$ ) nos diferentes tratamentos foi analisada pela análise de agrupamento (Cluster) por ligação completa, utilizando o programa STATISTICA (Statsoft, 1996). Tomou-se a distância mediana entre o número de organismos $\mathrm{m}^{-2}$ de cada grupo da macrofauna como medida de similaridade da média das três épocas de amostragens. A abundância (organismos por $\mathrm{m}^{2}$ ) dos principais grupos e, ou, ordens da macrofauna também foi transformada para freqüência relativa (\%). A freqüência foi avaliada de duas formas: freqüência relativa das ordens em cada um dos tratamentos; e freqüência relativa de cada ordem nos tratamentos. 
Quadro 1. Características químicas do dejeto de suínos usado (A) e do solo (B), na profundidade de 0-10 cm, referentes aos tratamentos estudados, na região de Campos Novos, SC

\begin{tabular}{|c|c|c|c|c|c|c|c|c|c|}
\hline & $\mathrm{pH} \mathrm{H}_{2} \mathrm{O}$ & $\mathbf{K}$ & $\mathbf{P}$ & $\mathbf{C a}$ & $\mathrm{Mg}$ & $\mathbf{N}$ & $\mathbf{M S}^{(1)}$ & $\mathbf{C u}$ & $\mathrm{Zn}_{\mathbf{n}}$ \\
\hline & & & 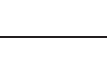 & $-\mathrm{g} \mathrm{k}$ & & & - & \multicolumn{2}{|c|}{$-\mathrm{mg} \mathrm{kg}^{-1}-$} \\
\hline Dejeto (A) & 7,8 & 20 & 27 & 18 & 0,9 & 32 & 56 & 8,8 & 32 \\
\hline Tratamento (B) & & \multicolumn{2}{|c|}{$-\mathrm{mg} \mathrm{kg}^{-1}-$} & \multicolumn{2}{|c|}{$-\mathrm{cmol}_{\mathrm{c}} \mathrm{dm}^{-3}-$} & & & & \\
\hline $\mathrm{TT}^{(2)}$ & 5,6 & 85 & 4,54 & 6,0 & 3,4 & (8) & - & - & - \\
\hline $\mathrm{AM}^{(3)}$ & 5,6 & 207 & 8,99 & 6,3 & 2,9 & - & - & - & - \\
\hline $\mathrm{AOM}^{(4)}$ & 5,7 & 315 & 19,53 & 7,0 & 3,0 & - & - & - & \\
\hline $\mathrm{AO} 3^{(5)}$ & 5,7 & 417 & 22,73 & 6,1 & 2,9 & - & - & - & - \\
\hline $\mathrm{AO}^{(6)}$ & 5,5 & 264 & 21,47 & 8,1 & 3,7 & - & - & - & 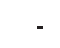 \\
\hline $\mathrm{AO} 12^{7)}$ & 5,8 & 370 & 45,27 & 8,3 & 3,7 & - & - & - & - \\
\hline
\end{tabular}

(1) MS: matéria seca. ${ }^{(2)}$ TT: testemunha. ${ }^{(3)}$ AM: adubação mineral. ${ }^{(4)}$ AOM: adubação organomineral. ${ }^{(5)}$ AO3: adubação orgânica com $3 \mathrm{t} \mathrm{ha}^{-1}$. ${ }^{(6)} \mathrm{AO6}$ : adubação orgânica com $6 \mathrm{t} \mathrm{ha}^{-1}$. ${ }^{(7)} \mathrm{AO} 12$ : adubação orgânica com $12 \mathrm{t} \mathrm{ha}^{-1}$. ${ }^{(8)}$ vValor não obtido.

\section{RESULTADOS E DISCUSSÃO}

\section{Freqüência relativa dos principais grupos da macrofauna edáfica}

Avaliando a freqüência relativa dos grupos da macrofauna em cada tratamento, observa-se que na primeira amostragem o tratamento TT obteve $83 \%$ de Hymenoptera (Quadro 2). Este tratamento foi o que teve o menor desenvolvimento das plantas (avaliação visual), devido ao não recebimento de adubação durante todo o período do estudo. Yang et al. (2007), estudando áreas fertilizadas ou não, com diferentes produções de massa verde, observaram relação linear direta entre produção de massa verde e abundância da fauna do solo. A menor produção de matéria seca por área pode inferir também uma menor disponibilidade de substrato para a macrofauna, que é muito sensível à variação da qualidade e quantidade das plantas de cobertura de solo (Lavelle et al., 1992). Dessa forma, pode-se esperar que essa alta freqüência de Hymenoptera esteja relacionada com a grande facilidade de locomoção desta ordem (Parr et al., 2007), que, em sua maioria, são Formicidae (formigas). Alves et al. (2006) encontraram valores semelhantes em áreas com diferentes sistemas de uso do solo, sobretudo nos tratamentos com maior revolvimento do solo e menor aporte de resíduos vegetais na sua superfície.

Ainda na primeira época de amostragem, o tratamento AM apresentou grande freqüência de Oligochaeta, representando mais de $87 \%$ da freqüência relativa total (Quadro 2). O tratamento AOM foi mais homogêneo em termos de distribuição da freqüência relativa, não apresentando ordens com tanta discrepância como outros tratamentos, conseqüência de uma adubação mais equilibrada, que beneficiou o crescimento das plantas e, conseqüentemente, a macrofauna do solo. Já o tratamento com a dose intermediária de adubação orgânica (AO6) apresentou alta freqüência de Oligochaeta (53 \%) (Quadro 2), o que não foi verificado nos tratamentos AO3 e AO12, nos quais a ordem Hymenoptera foi mais freqüente. Esse valor pode ser resultado da pouca quantidade de cobertura do solo, pois no tratamento $\mathrm{AO} 12$ foi adicionada uma dosagem muito alta de adubação orgânica, o que talvez possa ter afetado negativamente o desenvolvimento de algumas ordens e das plantas de cobertura, pela nãogerminação de algumas sementes (observação visual). A alta dose de adubação orgânica (AO12) também resultou em baixa produtividade na safra de 2004/ 2005 (dados não demonstrados). De modo geral, observou-se que a alta freqüência de Formicidae (formiga) pode ser resultado de áreas mal manejadas, o que é fundamental em estudos de impacto ambiental (Wink et al., 2005; Parr et al., 2007). Elas podem atuar na redistribuição das partículas, no transporte de nutrientes e na dinâmica da matéria orgânica, melhorando, assim, a infiltração de água no solo, pela melhor porosidade e aeração (Bruyn, 1999), além de serem citadas como indicadoras de alterações no manejo do solo, especialmente durante a sua reabilitação (Baretta et al., 2006; Parr et al., 2007).

Na segunda época de amostragem, novamente no tratamento TT, a ordem Hymenoptera foi a mais freqüente, mas com menor freqüência (40 \%) quando comparada com a anterior (Quadro 2). Esse decréscimo na freqüência de Hymenoptera pode ser devido à melhor qualidade de cobertura do solo, aumentando assim a freqüência das demais ordens (Parr et al., 2007). O tratamento AM apresentou menor ocorrência de Oligochaeta, sendo o grupo mais 
freqüente, com aproximadamente $66 \%$ do total. Esse alto valor pode ser devido à maior cobertura do solo, pois nesta época a área estava coberta com aveia, proporcionando melhor ambiente e disponibilidade de recursos para a macrofauna. No tratamento AOM, a ordem mais freqüente novamente foi Oligochaeta, representando mais de $86 \%$ do total de organismos. Nos tratamentos em que houve adição de adubação orgânica, novamente encontrou-se alta freqüência do grupo Oligochaeta, variando de 29 a $75 \%$ do total de organismos (Quadro 2). No tratamento AO3, a maior freqüência foi da ordem Hymenoptera, com aproximadamente $50 \%$ da freqüência total, com menor freqüência de Oligochaeta, possivelmente pela baixa dosagem do dejeto aplicado ao solo, resultando em menor desenvolvimento vegetativo da aveia em razão da disponibilidade de nutrientes para as plantas.
Na terceira amostragem, foram observados $21 \%$ de Hymenoptera no tratamento TT, valor este inferior ao das amostragens anteriores (Quadro 2), enquanto Oligochaeta foi o grupo mais freqüente, representando $34 \%$ da freqüência total, demonstrando que a época de amostragem influenciou a freqüência das ordens. No tratamento AM, a ordem mais presente foi Coleóptera, com $60 \%$ do total. A alta freqüência desta ordem pode ser efeito da cultura que estava implantada no momento da amostragem (milho). Algumas espécies de Coleoptera na fase larval podem ser consideradas pragas, conforme sua abundância e dano causados à cultura (Baretta et al., 2003). No AOM, obteve-se alta freqüência de Hymenoptera e Coleoptera, com 45 e $37 \%$ da freqüência total, respectivamente. No tratamento AO3, verificou-se mais alta freqüência para a ordem Coleoptera (53\%), seguida da ordem

Quadro 2. Freqüência relativa (\%) dos principais grupos da macrofauna do solo encontrados em cada tratamento e nas três épocas de amostragens

$$
\text { Tratamento Hymenoptera Coleóptera Oligochaeta Díptera Araneae Chilopoda Outros }{ }^{(1)}
$$

\begin{tabular}{|c|c|c|c|c|c|c|c|}
\hline \multicolumn{8}{|c|}{ Primeira amostragem $(5 / 5 / 2005)$} \\
\hline $\mathrm{TT}^{(2)}$ & 83,3 & 2,5 & 13,4 & 0 & 0,8 & 0 & 0 \\
\hline $\mathrm{AM}^{(3)}$ & 2,5 & 5,0 & 87,5 & 0 & 2,5 & 0 & 2,5 \\
\hline $\mathrm{AOM}^{(4)}$ & 24,3 & 30,0 & 24,3 & 0 & 11,4 & 8,6 & 1,4 \\
\hline $\mathrm{AO} 3^{(5)}$ & 66,7 & 14,0 & 15,7 & 1,8 & 0,9 & 0,9 & 0 \\
\hline $\mathrm{AO} 6^{(6)}$ & 8,5 & 25,5 & 53,3 & 0 & 8,5 & 2,1 & 2,1 \\
\hline $\mathrm{AO} 12^{(7)}$ & 60,2 & 11,8 & 12,9 & 1,1 & 2,2 & 7,5 & 4,3 \\
\hline \multicolumn{8}{|c|}{ Segunda amostragem $(9 / 9 / 2005)$} \\
\hline $\mathrm{TT}^{(2)}$ & 40,4 & 13,8 & 37,3 & 2,1 & 2,1 & 0 & 4,3 \\
\hline $\mathrm{AM}^{(3)}$ & 23,8 & 3,2 & 66,6 & 0 & 1,6 & 1,6 & 3,2 \\
\hline $\mathrm{AOM}^{(4)}$ & 4,9 & 4,2 & 85,5 & 2,4 & 0 & 1,2 & 1,8 \\
\hline $\mathrm{AO} 3^{(5)}$ & 49,1 & 15,8 & 29,8 & 0 & 0 & 1,8 & 3,5 \\
\hline $\mathrm{AO} 6^{(6)}$ & 3,4 & 15,4 & 75,1 & 2,7 & 0,7 & 1,3 & 1,4 \\
\hline $\mathrm{AO} 12^{(7)}$ & 14,8 & 24,1 & 29,7 & 24,1 & 1,8 & 0 & 5,5 \\
\hline \multicolumn{8}{|c|}{ Terceira Amostragem (23/1/2006) } \\
\hline $\mathrm{TT}^{(2)}$ & 21,0 & 31,6 & 34,2 & 2,6 & 5,3 & 0 & 5,3 \\
\hline $\mathrm{AM}^{(3)}$ & 26,9 & 61,5 & 3,8 & 0 & 3,9 & 3,9 & 0 \\
\hline $\mathrm{AOM}^{(4)}$ & 45,1 & 37,8 & 4,9 & 0 & 2,4 & 4,9 & 4,9 \\
\hline $\mathrm{AO} 3^{(5)}$ & 36,8 & 52,6 & 3,5 & 3,5 & 1,8 & 1,8 & 0 \\
\hline $\mathrm{AO} 6^{(6)}$ & 44,4 & 33,3 & 5,6 & 0 & 13,9 & 0 & 2,8 \\
\hline $\mathrm{AO} 12^{(7)}$ & 13,6 & 54,9 & 19,6 & 2,0 & 2,0 & 5,9 & 2,0 \\
\hline \multicolumn{8}{|c|}{ Média das três épocas de amostragens } \\
\hline $\mathrm{TT}^{(2)}$ & 58,0 & 11,0 & 25,4 & 1,2 & 2,0 & 0 & 2,4 \\
\hline $\mathrm{AM}^{(3)}$ & 17,8 & 15,6 & 60,5 & 0 & 2,3 & 1,5 & 2,3 \\
\hline $\mathrm{AOM}^{(4)}$ & 19,5 & 18,6 & 51,1 & 1,3 & 3,2 & 3,8 & 2,5 \\
\hline $\mathrm{AO} 3^{(5)}$ & 54,8 & 24,1 & 16,3 & 1,7 & 0,9 & 1,3 & 0,9 \\
\hline $\mathrm{AO} 6^{(6)}$ & 10,8 & 20,3 & 59,9 & 1,7 & 4,3 & 1,3 & 1,7 \\
\hline $\mathrm{AO} 12^{(7)}$ & 35,9 & 26,3 & 19,2 & 7,6 & 2,0 & 5,0 & 4,0 \\
\hline
\end{tabular}

(1) Outros: somatório de organismos com menor freqüência. ${ }^{(2)}$ TT: testemunha. ${ }^{(3)}$ AM: adubação mineral. ${ }^{(4)}$ AOM: adubação organomineral. ${ }^{(5)}$ AO3: adubação orgânica com 3 t ha $^{-1}{ }^{(6)}$ AO6: adubação orgânica com 6 t ha $^{-1}$. ${ }^{(7)}$ AO12: adubação orgânica com $12 \mathrm{t} \mathrm{ha}^{-1}$. 
Hymenoptera (37\%). Algumas espécies da família Carabidae (Coleoptera) são sensíveis indicadores de variação de temperatura e umidade do solo (Stork \& Eggleton, 1992). Ainda nesta amostragem, os tratamentos com aplicação de adubação orgânica, independentemente da dose, apresentaram baixa freqüência de Oligochaeta, possivelmente em razão do grande período de estiagem, pois este grupo é sensível às variações de umidade do solo (Silva et al., 2006; Baretta et al., 2007).

Considerando a média das três épocas de amostragens, evidenciou-se que a ordem Hymenoptera foi a mais freqüente, sobretudo nos tratamentos TT, $\mathrm{AO} 3$ e AO12, com aproximadamente 58, 56 e $36 \%$ da freqüência total de organismos, respectivamente (Quadro 2). Esse comportamento pode ter ocorrido em razão da baixa cobertura do solo e da sua baixa fertilidade, especialmente nos tratamentos TT e AO3 (Quadro 1), refletindo em pouco substrato para os outros grupos da macrofauna (Warren \& Zou, 2002) e, conseqüentemente, a dominância das formigas sobre as demais ordens, especialmente pela facilidade de locomoção (Parr et al., 2007). Estudos mostram que o sistema de preparo convencional do solo apresenta, em relação ao plantio direto, uma predominância de Hymenoptera, sobretudo por apresentar menor cobertura e maior revolvimento do solo (Alves et al., 2006; Baretta et al., 2006). As minhocas (Oligochaeta) foram mais freqüentes nos tratamentos AM, AOM e AO6, com mais de $50 \%$ da ocorrência total de organismos, confirmando os estudos de Pandolfo et al. (2005), que encontraram alta freqüência desta ordem onde foram aplicadas fontes de nutrientes de origem orgânica. É importante salientar que, no tratamento AM, mais de $60 \%$ do total de organismos foi de Oligochaeta; esse alto valor pode ser devido à adição de fertilizantes minerais ao solo, o que proporcionou maior produção de massa vegetal (Edwards \& Bohlen, 1996). Outro destaque nos tratamentos AOM, AO3, AO6 e AO12 é a ordem Coleóptera, com variação de 18 a $26 \%$ (Quadro 2). Segundo Thomanzini \& Thomanzini (2002), algumas famílias de Coleóptera - por exemplo, os escarabeídeos (Escarabeidae) - alimentam-se de fezes e carcaças oriundas de vertebrados, e isso pode ter influenciado a alta freqüência da ordem Coleoptera nos tratamentos em que foram adicionados dejetos de suínos.

A avaliação da freqüência de ordens entre amostragens (Quadro 3) apontou a ordem Hymenoptera como a mais freqüente nos tratamentos TT e AO3 nas duas primeiras amostragens. Já na terceira amostragem, a maior freqüência de Hymenoptera foi encontrada no tratamento AOM. Essa variação na freqüência pode ter sido ocasionada pela grande estiagem na terceira época de amostragem, diminuindo assim a freqüência dos demais grupos da macrofauna nos tratamentos. Menor umidade associada à menor fertilidade, nos tratamentos TT e AO3, pode diminuir a disponibilidade de alimento, visto que nestes tratamentos foi observado menor desenvolvimento da parte aérea da cultura do milho e da aveia, o que proporcionou menor rendimento de grãos (dados não demonstrados). As demais ordens variam entre as épocas de amostragens, e Coleoptera e a Diptera (não edáfico) predominaram nos tratamentos com aplicação de dejeto de suínos. A maior freqüência de Chilopoda foi observada nos tratamentos com adição de dejetos e com melhor fertilidade (Quadro 1), confirmando resultados de Dindal (1990), que verificou acréscimo da freqüência de alguns grupos da fauna edáfica, especialmente onde o solo se encontrava em condições de melhor umidade e fertilidade. No tratamento TT não foi encontrado Chilopoda; este grupo é muito dependente da quantidade e da qualidade da cobertura do solo (Almeida et al., 2007), o que pode explicar a ausência deste organismo neste tratamento (Quadro 3).

Considerando as três épocas de amostragens (Quadro 3), as minhocas (Oligochaeta) tiveram a maior freqüência no tratamento $\mathrm{AOM}$, confirmando estudos de Aquino (1994), em que foi utilizado um composto organomineral de esterco bovino com bagaço de cana-de-açúcar e adubo químico. A metade da freqüência relativa total (50 \%) de Diptera (não edáfico) foi encontrada no tratamento com maior adição de dejeto suíno (AO12), embora esta ordem no tratamento $\mathrm{AM}$, o que demonstra claramente que a adição de adubação orgânica foi benéfica ao seu desenvolvimento e a adição da adubação mineral foi prejudicial. As aranhas (Araneae) tiveram alta freqüência no tratamento AOM (aproximadamente $30 \%$ do total) e a menor no tratamento com a maior dosagem de dejeto AO12 (menos de $2 \%$ do total), sendo este grupo afetado pela alta dosagem de dejeto.

\section{Diversidade da macrofauna edáfica}

$\mathrm{Na}$ primeira época de amostragem não houve diferença significativa para a abundância e riqueza total de ordens encontradas (Quadro 4). Os maiores valores de diversidade de Shanonn $(\mathrm{H})$, dominância de Simpson (Is) e uniformidade de Pielou (e) foram encontrados nos tratamentos AOM, AO6 e AO12, e maior valor no AO3. Os tratamentos TT e AM apresentaram os menores valores dos índices de diversidade ecológica. Apesar de apresentar baixa densidade no tratamento AOM, não se verificaram baixos valores dos índices, o que pode estar associado à adição de matéria orgânica pelo dejeto aplicado na área (Correia \& Pinheiro, 1999).

$\mathrm{Na}$ segunda amostragem, só o valor de Is apresentou diferença significativa. $\mathrm{O}$ alto valor de Is no tratamento TT se deve à alta dominância das ordens Oligochaeta e Hymenoptera, pois, com exceção do tratamento AO12, nos demais estas duas ordens juntas chegaram a representar mais de $75 \%$ da freqüência total da macrofauna encontrada (Quadro 2). $\mathrm{Na}$ terceira amostragem, os valores de uniformidade de Pielou (e) não mostraram diferenças significativas entre os tratamentos (Quadro 4). Verificou-se 
Quadro 3. Freqüência relativa (\%) de cada um dos principais grupos da macrofauna do solo nos tratamentos e nas três épocas de amostragens

\begin{tabular}{|c|c|c|c|c|c|c|c|}
\hline Tratamento & Hymenoptera & Coleóptera & Oligochaeta & Díptera & Araneae & Chilopoda & Outros $^{(1)}$ \\
\hline & \multicolumn{7}{|c|}{ Primeira amostragem $(5 / 5 / 2005)$} \\
\hline $\mathrm{TT}^{(2)}$ & 39,4 & 4,6 & 13 & 0 & 5,9 & 0 & 0 \\
\hline $\mathrm{AM}^{(3)}$ & 0,4 & 3,1 & 28,5 & 0 & 5,9 & 0 & 14,3 \\
\hline $\mathrm{AOM}^{(4)}$ & 6,7 & 32,3 & 13,8 & 0 & 47 & 40 & 14,3 \\
\hline $\mathrm{AO} 3^{(5)}$ & 29,9 & 24,6 & 14,6 & 66,7 & 5,9 & 6,6 & 0 \\
\hline $\mathrm{AO}^{(6)}$ & 1,6 & 18,5 & 20,3 & 0 & 23,5 & 6,7 & 14,3 \\
\hline \multirow[t]{2}{*}{$\mathrm{AO} 12^{(7)}$} & 22 & 16,9 & 9,8 & 33,3 & 11,8 & 46,7 & 57,1 \\
\hline & \multicolumn{7}{|c|}{ Segunda amostragem $(9 / 9 / 2005)$} \\
\hline $\mathrm{TT}^{(2)}$ & 37,3 & 19,4 & 9,6 & 8,7 & 40,0 & 0 & 25,0 \\
\hline $\mathrm{AM}^{(3)}$ & 14,7 & 3,0 & 11,6 & 0 & 20,0 & 16,7 & 12,5 \\
\hline $\mathrm{AOM}^{(4)}$ & 7,8 & 10,5 & 38,8 & 17,4 & 0 & 33,3 & 18,7 \\
\hline $\mathrm{AO} 3^{(5)}$ & 27,4 & 13,4 & 4,7 & 0,0 & 0 & 16,7 & 12,5 \\
\hline $\mathrm{AO} 6^{(6)}$ & 5,0 & 34,3 & 30,9 & 17,4 & 20,0 & 33,3 & 12,5 \\
\hline \multirow[t]{2}{*}{$\mathrm{AO} 12^{(7)}$} & 7,8 & 19,4 & 4,4 & 56,5 & 20,0 & 0 & 18,8 \\
\hline & \multicolumn{7}{|c|}{ Terceira amostragem $(23 / 1 / 2006)$} \\
\hline $\mathrm{TT}^{(2)}$ & 8,3 & 9,3 & 40,6 & 25,0 & 16,7 & 0 & 25,0 \\
\hline $\mathrm{AM}^{(3)}$ & 7,3 & 12,4 & 3,1 & 0 & 8,3 & 11,1 & 0 \\
\hline $\mathrm{AOM}^{(4)}$ & 38,5 & 24,0 & 12,5 & 0 & 16,7 & 44,5 & 50,0 \\
\hline $\mathrm{AO} 3^{(5)}$ & 21,9 & 23,3 & 6,3 & 50,0 & 8,3 & 11,1 & 0 \\
\hline $\mathrm{AO} 6^{(6)}$ & 16,7 & 9,3 & 6,3 & 0 & 41,7 & 0 & 12,5 \\
\hline \multirow[t]{2}{*}{$\mathrm{AO} 12^{(7)}$} & 7,3 & 21,7 & 31,2 & 25,0 & 8,3 & 33,3 & 12,5 \\
\hline & \multicolumn{7}{|c|}{ Média das amostragens } \\
\hline $\mathrm{TT}^{(2)}$ & 32,3 & 10,7 & 12,3 & 10,0 & 14,7 & 0 & 19,3 \\
\hline $\mathrm{AM}^{(3)}$ & 5,1 & 7,6 & 15,1 & 0,0 & 8,8 & 6,7 & 9,7 \\
\hline $\mathrm{AOM}^{(4)}$ & 13,7 & 22,6 & 31,3 & 13,3 & 29,4 & 40,0 & 25,8 \\
\hline $\mathrm{AO} 3^{(5)}$ & 27,7 & 21,2 & 7,2 & 13,3 & 5,9 & 10,0 & 6,5 \\
\hline $\mathrm{AO} 6^{(6)}$ & 5,5 & 18,0 & 26,8 & 13,4 & 29,4 & 10,0 & 12,9 \\
\hline $\mathrm{AO} 12^{(7)}$ & 15,7 & 19,9 & 7,3 & 50,0 & 11,8 & 33,3 & 25,8 \\
\hline
\end{tabular}

(1) Outros: somatório de organismos com menor freqüência. ${ }^{(2)}$ TT: testemunha. ${ }^{(3)}$ AM: adubação mineral. ${ }^{(4)}$ AOM: adubação organomineral. ${ }^{(5)} \mathrm{AO} 3$ : adubação orgânica com $3 \mathrm{t} \mathrm{ha}^{-1} .{ }^{(6)} \mathrm{AO6}$ : adubação orgânica com $6 \mathrm{t} \mathrm{ha}^{-1}$. ${ }^{(7)} \mathrm{AO} 12$ : adubação orgânica com $12 \mathrm{tha}^{-1}$.

comportamento diferente nesta época, com menores valores de $\mathrm{H} \mathrm{e} \mathrm{Is,} \mathrm{no} \mathrm{tratamento} \mathrm{AO3} \mathrm{(Quadro} \mathrm{4).} \mathrm{Isso}$ possivelmente é resultante da alta freqüência das ordens Coleoptera e Hymenoptera neste tratamento, além do efeito de época e, ou, do tipo de cobertura do solo no período da amostragem. Por sua vez, o tratamento AOM apresentou maiores valores dos índices $\mathrm{H}$ e abundância, sendo o mais estável e evidenciando a melhor resposta à adição do dejeto junto à adubação mineral. Esta amostragem apresentou maiores valores dos índices de diversidade - reflexo da menor precipitação neste período. Resultados semelhantes foram encontrados em um estudo de monitoramento da fauna do solo em diferentes coberturas vegetais na área experimental do SIPA, no Rio de Janeiro, RJ (Correia \& Pinheiro, 1999).
Considerando a média das três épocas de amostragens, o índice de diversidade de Shannon (Quadro 4) variou de 0,6 (tratamento AM) a 1,1 (tratamento AOM), indicando efeito negativo sobre o desenvolvimento da macrofauna, sobretudo quando se utilizou somente a adubação mineral. Em um estudo com e sem adição de fertilizante mineral, Sileshi \& Mafongoya (2006) verificaram menor valor de diversidade no tratamento com fertilizante mineral, corroborando os resultados aqui apresentados. Por outro lado, quando se associou fertilizante mineral com o orgânico (AOM), verificou-se efeito benéfico sobre a macrofauna. No tratamento com dose mais baixa (AO3) de adubação orgânica a diversidade não foi influenciada, alcançando valores próximos aos do tratamento TT. Os tratamentos AO6 e AO12 apresentaram altos valores de $\mathrm{H}$ quando comparados 
Quadro 4. Valores dos índices de diversidade de Shanonn (H), dominância de Simpson (Is), uniformidade de Pielou (e), riqueza de grupos (total de ordens encontradas) e da abundância (Organismos $\mathrm{m}^{-2}$ ) da macrofauna edáfica nos seis tratamentos estudados e nas três épocas de amostragens

\begin{tabular}{|c|c|c|c|c|c|}
\hline Tratamento & $\mathbf{H}$ & Is & $\boldsymbol{e}$ & Riqueza & Org $\mathbf{~}^{-2}$ \\
\hline & \multicolumn{5}{|c|}{ Primeira amostragem $(5 / 5 / 2005)$} \\
\hline $\mathrm{TT}^{(1)}$ & $0,3 \mathrm{c}$ & $0,2 \mathrm{c}$ & $0,7 \mathrm{~b}$ & $5^{*}$ & 320,0 * \\
\hline $\mathrm{AM}^{(2)}$ & $0,5 \mathrm{bc}$ & $0,4 \mathrm{c}$ & $0,8 \mathrm{~b}$ & 7 & 106,6 \\
\hline $\mathrm{AOM}^{(3)}$ & $1,2 \mathrm{a}$ & $0,7 \mathrm{ab}$ & $2,0 \mathrm{a}$ & 8 & 186,6 \\
\hline $\mathrm{AO}^{(4)}$ & $0,6 \mathrm{bc}$ & $0,5 \mathrm{bc}$ & $1,3 \mathrm{ab}$ & 8 & 304,0 \\
\hline $\mathrm{AO}^{(5)}$ & $1,1 \mathrm{a}$ & $0,9 \mathrm{a}$ & $1,8 \mathrm{a}$ & 8 & 125,3 \\
\hline \multirow[t]{2}{*}{$\mathrm{AO} 12^{(6)}$} & $0,9 \mathrm{ab}$ & $0,7 \mathrm{ab}$ & $1,5 \mathrm{ab}$ & 9 & 248,0 \\
\hline & \multicolumn{5}{|c|}{ Segunda amostragem (9/9/2005) } \\
\hline $\mathrm{TT}^{(1)}$ & $0,8^{*}$ & $0,6 \mathrm{a}$ & $1,6 *$ & $9 *$ & $250,7^{*}$ \\
\hline $\mathrm{AM}^{(2)}$ & 0,5 & $0,3 \mathrm{~b}$ & 1,1 & 8 & 168,0 \\
\hline $\mathrm{AOM}^{(3)}$ & 1,2 & $0,4 \mathrm{ab}$ & 1,4 & 9 & 440,0 \\
\hline $\mathrm{AO}^{(4)}$ & 0,7 & $0,5 \mathrm{ab}$ & 1,6 & 5 & 152,0 \\
\hline $\mathrm{AO} 6^{(5)}$ & 1,1 & $0,4 \mathrm{ab}$ & 1,4 & 10 & 397,3 \\
\hline \multirow[t]{2}{*}{$\mathrm{AO} 12^{(6)}$} & 0,9 & $0,5 \mathrm{ab}$ & 1,5 & 9 & 144,0 \\
\hline & \multicolumn{5}{|c|}{ Terceira amostragem $(23 / 1 / 2006)$} \\
\hline $\mathrm{TT}^{(1)}$ & $1,2 \mathrm{ab}$ & $0,8 \mathrm{a}$ & $1,3^{*}$ & $8 \mathrm{a}$ & $101,3 \mathrm{~b}$ \\
\hline $\mathrm{AM}^{(2)}$ & $0,9 \mathrm{abc}$ & $0,8 \mathrm{a}$ & 1,1 & $6 \mathrm{~b}$ & $69,3 \mathrm{~b}$ \\
\hline $\mathrm{AOM}^{(3)}$ & $1,3 \mathrm{a}$ & $0,7 \mathrm{ab}$ & 1,9 & $8 \mathrm{a}$ & 218,7 a \\
\hline $\mathrm{AO}^{(4)}$ & $0,8 \mathrm{c}$ & $0,5 \mathrm{~b}$ & 1,4 & $8 \mathrm{a}$ & $152,0 \mathrm{ab}$ \\
\hline $\mathrm{AO} 6^{(5)}$ & $1,0 \mathrm{abc}$ & $0,7 \mathrm{ab}$ & 1,6 & $6 \mathrm{~b}$ & $96,0 \mathrm{~b}$ \\
\hline \multirow[t]{2}{*}{$\mathrm{AO} 12^{(6)}$} & $0,8 \mathrm{c}$ & $0,6 \mathrm{ab}$ & 1,6 & $8 \mathrm{a}$ & $136,0 \mathrm{ab}$ \\
\hline & \multicolumn{5}{|c|}{ Médias das Amostragens } \\
\hline $\mathrm{TT}^{(1)}$ & $0,8 \mathrm{bc}$ & $0,5 \mathrm{~b}$ & $1,2 \mathrm{bc}$ & $7,33^{*}$ & $224,0 \mathrm{ab}$ \\
\hline $\mathrm{AM}^{(2)}$ & $0,6 \mathrm{c}$ & $0,5 \mathrm{~b}$ & $1,0 \mathrm{c}$ & 7,00 & $114,7 \mathrm{~b}$ \\
\hline $\mathrm{AOM}^{(3)}$ & $1,1 \mathrm{a}$ & $0,6 \mathrm{ab}$ & $1,8 \mathrm{a}$ & 8,33 & $281,8 \mathrm{a}$ \\
\hline $\mathrm{AO}^{(4)}$ & $0,7 \mathrm{bc}$ & $0,5 \mathrm{~b}$ & $1,4 \mathrm{abc}$ & 7,00 & $202,7 \mathrm{ab}$ \\
\hline $\mathrm{AO} 6^{(5)}$ & $1,0 \mathrm{ab}$ & $0,7 \mathrm{a}$ & $1,6 \mathrm{ab}$ & 8,00 & $206,2 \mathrm{ab}$ \\
\hline $\mathrm{AO} 12^{(6)}$ & $0,9 \mathrm{ab}$ & $0,6 a b$ & $1,6 \mathrm{ab}$ & 8,67 & $176,0 \mathrm{ab}$ \\
\hline
\end{tabular}

(1) TT: testemunha. ${ }^{(2)} \mathrm{AM}$ : adubação mineral. ${ }^{(3)} \mathrm{AOM}$ : adubação dejeto + mineral. ${ }^{(4)} \mathrm{AO}$ : adubação orgânica com 3 t ha ${ }^{-1} .{ }^{(5)} \mathrm{AO} 6$ :

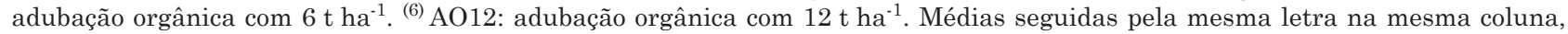
não diferem entre si pelo teste de LSD a $5 \%$;: Não-significativo (LSD a $5 \%$ ).

com os tratamentos AM e TT, sugerindo que as dosagens maiores podem ter beneficiado a diversidade da macrofauna edáfica. Já o índice de Simpson variou de 0,5 a 0,7 . Os menores valores de Is foram obtidos nos tratamentos TT, AM e AO3. O maior valor de Is foi encontrado no tratamento AO6 $(0,7)$, possivelmente pelo alto valor de Oligochaeta (Quadro 2). O valor da uniformidade de Pielou (e) variou de 1,0 a 1,8, sendo o tratamento AM o mais desuniforme e o tratamento AOM o mais uniforme, seguidos pelo AO6 e AO12, demonstrando que o dejeto utilizado em conjunto com adubação mineral foi benéfico para a macrofauna (Quadro 4).

A abundância da macrofauna, quando avaliadas as médias das três amostragens (Quadro 4), variou de 115 (AM) a 282 org m$^{-2}(\mathrm{AOM})$; o tratamento AM obteve os menores valores, demonstrando que a macrofauna é sensível a este tipo de adubação. A adição de fertilizantes minerais pode ser tóxica a alguns organismos da fauna do solo, como a intoxicação das minhocas pela amônia (Kladivko \& Timmenga, 1990). Já a adição de adubos orgânicos pode ter efeito benéfico sobre alguns grupos da macrofauna de solo, como demonstrado por Edwards \& Lofty (1982); Baretta et al. (2003), pois, além do maior aporte de nutrientes ao solo, representa fonte alimentar adicional para os organismos edáficos (Kladivko \& Timmenga, 1990).

\section{Análise de agrupamento para macrofauna edáfica}

A análise de agrupamento evidencia a similaridade entre os tratamentos em relação à abundância da macrofauna edáfica por meio da distância mediana entre os números de organismos capturados por $\mathrm{m}^{2}$ de cada grupo ou classe taxonômica. 
Os tratamentos AO3 e TT foram similares entre si e formaram um agrupamento separado dos demais. Outro agrupamento foi formado entre os demais tratamentos, especialmente entre $\mathrm{AM}$ e $\mathrm{AO} 12$, e entre AO6 e AOM (Figura 1). Este último agrupamento $(\mathrm{AO} 6$ e $\mathrm{AOM})$, isso pode ser resultado da resposta da macrofauna à alta quantidade de dejetos adicionada. Estudos mostram que alguns nutrientes em excesso apresentam efeito tóxico sobre alguns grupos da macrofauna - o mesmo pode ser atribuído à adubação química (Edwards \& Lofty, 1982; Yang et al., 2007). Já a similaridade encontrada nos tratamentos em que não houve adubação nenhuma (TT) e naqueles em que a adubação orgânica esteve em baixa quantidade (AO3) pode ser explicada pela baixa quantidade de nutrientes incorporados ao solo e sua menor disponibilidade às plantas. Venturini (2003) encontrou resultados semelhantes quando estudou dosagens de vermicomposto sobre a macrofauna, e o tratamento com adubação química também foi similar ao de menor dosagem do vermicomposto.

Quanto ao arranjamento dos grupos da macrofauna, evidenciou-se que a ordem Hymenoptera se isolou das demais, seguida por Oligochaeta, Coleoptera, Diptera (não edáfico), Araneae e Chilopoda. Esses resultados corroboram os de Rovedder et al. (2004), que observaram o mesmo com a ordem Hymenoptera. Esse comportamento foi explicado pelos autores por meio da freqüência relativa desta ordem. Os demais grupos - Lepidóptera, Dermaptera, Blattodea, Outros, Mollusca, Hemiptera e Isoptera - apresentaram menores valores de distância de ligação e foram muito similares entre si. Esse comportamento está relacionado com a maior ou menor abundância de cada grupo.

\section{CONCLUSÕES}

1. A freqüência relativa das ordens mais freqüentes (Hymenoptera, Oligochaeta e Coleoptera) foi afetada pela adição da adubação orgânica com dejeto de suínos, sendo o comportamento dependente da época de amostragem.

2. A maior diversidade da macrofauna do solo foi encontrada no tratamento com adubação organomineral, demonstrando que a macrofauna edáfica foi beneficiada pela adição do fertilizante mais balanceado.

3 . Sugere-se que a quantidade e qualidade da cobertura do solo e, ou, palhada também influenciou a abundância e a diversidade da macrofauna do solo.

\section{LITERATURA CITADA}

ALMEIDA, H.C.; ALMEIDA, D.; ALVES, M.V.; SCHNEIDER, J.; MAFRA, Á.L. \& BERTOL, I. Propriedades químicas e fauna do solo influenciadas pela calagem em sistema semeadura direta. Ci. Rural, 37:1462-1465, 2007.

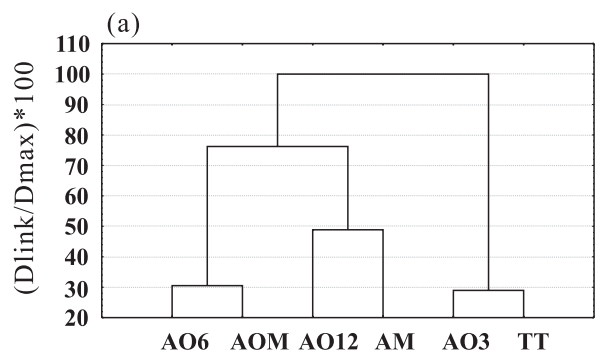

(b)

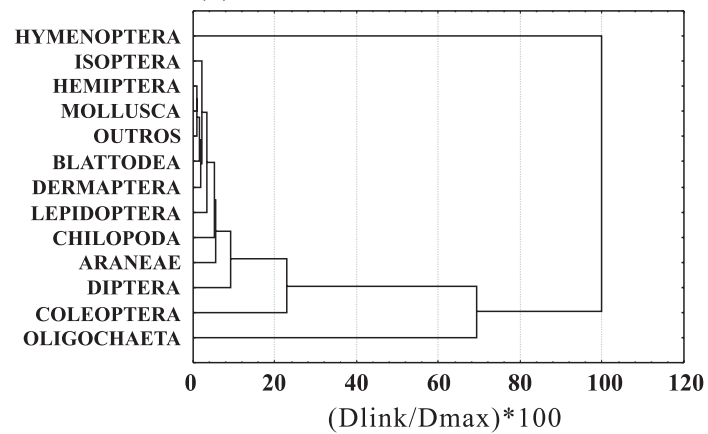

Figura 1. Dendrograma apresentando as distâncias de ligação entre os tratamentos (a), por meio da abundância dos grupos (organismos por $\mathrm{m}^{2}$ ) da macrofauna edáfica na profundidade de $0-10 \mathrm{~cm}$ (b). Média de seis repetições e três épocas de coleta. TT: testemunha; AM: adubação mineral; AOM: adubação organomineral; AO3: adubação orgânica com 3 t ha' h $^{-1}$ AO6: adubação orgânica com 6 t ha $^{-1}$; A012: Adubação orgânica com 12 t ha ${ }^{-1}$. Outros: somatório de organismos com menor freqüência.

ALVES, M.V.; BARETTA, D. \& CARDOSO, E.B.J. Fauna edáfica em diferentes sistemas de cultivo no estado de São Paulo. R. Ci. Agrovet., 5:31-41, 2006.

ANDERSEN, A. Plant protection in spring cereal production with reduced tillage. II Pests and beneficial insects. Crop Protec., 18:651-657, 1999.

AQUINO, A.M. Reprodução de minhocas (Oligochaeta) em dejeto suíno e bagaço de cana-de-açúcar. Pesq. Agropec. Bras., 19:161-168, 1994

BARETTA, D. Atributos biológicos do solo em diferentes agroecossistemas na região sul do Brasil. Lages, Universidade do Estado de Santa Catarina, 2003. 123p. (Tese de Mestrado)

BARETTA, D.; BROWN, G.G.; JAMES, S.W. \& CARDOSO, E.J.B.N. Earthworm populations sampled using collection methods in Atlantic Forests with Araucaria angustifolia. Sci. Agric., 64:384-392, 2007.

BARETTA, D.; MAFRA, A.L.; SANTOS, J.C.P.; AMARANTE, C.V.T. \& BERTOL, I. Análise multivariada da fauna edáfica em diferentes sistemas de preparo e cultivo do solo. Pesq. Agropec. Bras., 41:1675-1679, 2006.

BARETTA, D.; SANTOS, J.C.P.; MAFRA, A.L.; WILDNER, L.P. \& MIQUELLUTI, D.J. Fauna edáfica avaliada por armadilhas de catação manual afetada pelo manejo do solo na região oeste catarinense. R. Ci. Agrovet., 2:97106, 2003 . 
BRUYN, L.A.L. Ants as bioindicators of soil function in rural environments. Agric., Ecosyst. Environ., 74:425-441, 1999.

COMISSÃO DE QUÍMICA E FERTILIDADE DO SOLO . CQFSRS/SC. Manual de adubação e de calagem para os estados do Rio Grande do Sul e Santa Catarina. 10.ed. Porto Alegre, SBCS/NRS, 2004. 400p.

CORREIA, M.E.F. \& PINHEIRO, L.B.A. Monitoramento da fauna do solo sob diferentes coberturas vegetais em um sistema integrado de produção agroecológica, Seropédica (RJ). Seropédica, Embrapa Agrobiologia, 1999. 15p. (Circular Técnica, 3)

DECAËNS, T.; JIMÉNEZ, J.J. \& LAVELLE, P. Effect of exclusion of the anecic earthworm Martiodrilus carimaguensis Jiménez and Moreno on soil properties and plant growth in grasslands of the eastern plains of Colombia. Pedobiologia, 43:835-841, 1999.

DINDAL, D.L. Soil biology guide. New York, John Wiley, 1990. 1349p.

EDWARDS, C.A. \& BOHLEN, P.J. Biology and ecology of earthworms. 3.ed. London, Chapman and Hall, 1996. 426p.

EDWARDS, C.A. \& LOFTY, J.R. Nitrogenous fertilizers and earthworms populations in agricultural soils. Soil Biol. Biochem., 14:515-521, 1982.

HENDRIX, P.F.; CROSSLEY JR., D.A.; BLAIR, J.M. \& COLEMAN, D.C. Soil biota as components of sustainable agroecosystems. In: EDWARDS, C.A.; LAL, R.; MADDEN, P.; MILLER, R.H. \& HOUSE, G. Sustainable agricultural systems. Ankey, Soil and Water Conservation Society, 1990. p.637-654.

INSTITUTO BRASILEIRO DE GEOGRAFIA E ESTATÍSTICA - IBGE. Indicadores IBGE - Estatística da Produção Pecuária. Rio de Janeiro, 2006. 24p.

KLADIVKO, E.J. \& TIMMENGA, H.J. Earthworms and agricultural management. In: BOX, J.E. \& HAMMOND, L.C., eds. Rhizosphere dynamics. Madison, American Society of Agronomy, 1990. p.192-216. (Selected Symposium, 113)

LAVELLE, P.; BLANCHART, E.; MARTIN, A.; SPAIN, A.V. \& MARTIN, S. The impact of soil fauna on the properties of soils in the humid tropics. In: SANCHEZ, P.A. \& LAL, R., eds. Myths and science of soils of the tropics. Madison, Soils Science Society of America, 1992. p.157-185 (Special Publ.)

MINER, J.R. Alternatives to minimize the enviromental impact of large swine production units. J. Anim. Sci., 77:440444, 1999.

ODUM, E.P. Ecologia. Rio de Janeiro, Guanabara, 1983. 434p.

OUÉDRAOGO, E.; MANDO, A. \& BRUSSAARD, L. Soil macrofauna affect crop nitrogen and water use efficiencies in semi-arid West Africa. Eur. J. Soil Biol., 42:275-277, 2006.

PANDOLFO, C.M.; CERETTA, C.A.; VEIGA, M. \& GIROTTO, E. Estudo da mesofauna edáfica em diferentes sistemas de manejo do solo e fontes de nutrientes. Agropec. Catarinense, 18:63-67, 2005.
PARR, C.L.; ANDERSEN, A.N.; CHASTAGNOL, A. \& DUFFAUD, C. Savanna fires increase rates and distances of seed dispersal by ants. Oecologia, 151:33-41, 2007.

ROVEDDER, A.P.; ANTONIOLL, Z.I.; SPAGNOLLO, E. \& VENTURINI, S.F. Fauna edáfica em solo suscetível à arenização na região sudoeste do rio grande do sul. R. Ci. Agrovet., 3:87-96, 2004.

SCHERER, E.E. \& CASTILHOS, E.G. Esterco de suínos como fonte de nitrogênio para milho e feijão da safrinha. Agropec. Catarinense, 7:25-28, 1994.

SEIFFERT, N.F. \& PERDOMO, C.C. Aptidão de solos da bacia hidrográfica do rio do peixe para aporte de fertilizantes orgânicos. Concórdia, Embrapa/CNPSA, 1998. (Comunicado Técnico, 230)

SILESHI, G. \& MAFONGOYA, P.L. Long-term effects of improved legume fallows on soil invertebrate macrofauna and maize yield in eastern Zambia. Agric. Ecosyst. Environ., 115:69-78, 2006.

SILVA, R.F.; AQUINO, A.M.; MERCANTE, F.M. \& GUIMARÃES, M.D.E.F. Populações de oligoquetos (Annelida: Oligochaeta) em um Latossolo Vermelho submetido a sistemas de uso do solo. Ci. Rural, 36:673677,2006 .

STATSOFT INC. Statistica for Windows. Computer program manual. Tulsa, 1996.

STORK, N.E. \& EGGLETON, P. Invertebrates as determinants and indicators of soil quality. Am. J. Altern. Agric., 7:3847, 1992.

TEDESCO, M.J.; GIANELLO, C.; BISSANI, C.A.; BOHNEN, H. \& VOLKWEISS, S.J. Análises de solo, plantas e outros materiais. 2.ed. Porto Alegre, Universidade Federal do Rio grande do Sul, 1995. 174p. (Boletim Técnico, 5)

THOMANZINI, M.J. \& THOMANZINI, A.P.B.W. Levantamento de insetos e análise entomofauna em florestas, capoeira e pastagem no Sudeste Acreano. Rio Branco, Embrapa, 2002. 41p. (Circular Técnica, 35)

VENTURINI, S.F. Efeito do uso de vermicomposto na população de organismos edáficos, nutrição e produção de grãos de feijoeiro. Santa Maria, Universidade Federal de Santa Maria, 2003. 67p. (Tese de Mestrado)

WARREN, M.W. \& ZOU, X. Soil macrofauna and litter nutrients in three plantations on a disturbed site in Puerto Rico. For. Ecol. Manag., 170:161-171, 2002.

WINK, C.; GUEDES, J.V.C.; FAGUNDES, C.K. \& ROVEDDER, A.P. Insetos edáficos como indicadores da qualidade ambiental. R. Ci.. Agrovet., 4:60-71, 2005.

YANG, X.; WARREN, M. \& ZOU, X. Fertilization responses of soil litter fauna and litter quantity, quality, and turnover in low and high elevation forests of Puerto Rico. Appl. Soil Ecol., 37:63-71, 2007. 